\title{
Correction to: Inexact rational Krylov method for evolution equations
}

\author{
Yuka Hashimoto $^{1}$ (D) Takashi Nodera ${ }^{2}$
}

Received: 2 August 2021 / Accepted: 27 August 2021 / Published online: 6 September 2021

(c) Springer Nature B.V. 2021

\section{Correction to: BIT Numerical Mathematics (2021) 61:473-502 https://doi.org/10.1007/s10543-020-00829-w}

\section{Correction of the statements and proofs of Theorem 3.1 and Proposition 3.1}

The proof of Proposition 3.1 is incorrect. First, the unitary matrix $Q_{m}$ in the proof does not transform $\tilde{K}_{m}$ into an upper Hessenberg matrix. For $j \geq 2$, the definition of $u_{j}$ has to be replaced by $u_{j}:=\left(\hat{k}_{j+1: m, j}^{j}-\eta_{j} e_{1}\right) /\left\|\hat{k}_{j+1: m, j}^{j}-\eta_{j} e_{1}\right\|$, where $\hat{k}_{j+1: m, j}^{j}$ is the vector consisting of the elements in rows $j+1 \sim m$ and column $j$ of the matrix $\hat{K}_{m, j}:=\left(I_{m}+\hat{Q}_{j}\right) \cdots\left(I_{m}+\hat{Q}_{2}\right) \tilde{K}_{m}\left(I_{m}+\hat{Q}_{2}\right) \cdots\left(I_{m}+\hat{Q}_{j}\right)$ and $\eta_{j}:=-\operatorname{sign}\left(\hat{k}_{j+1, j}\right)\left\|\hat{k}_{j+1: m, j}\right\|$. In addition, the sum $\sum_{k=3}^{\min \{i, j\}}$ in the formula after the equation (A.2) has to be replaced by $\sum_{k=3}^{i}$. Thus, the upper bound of $\left|\left(Q_{m}-I_{m}\right)_{i, j}\right|$ for $i>j$ is not the same as that for $i \leq j$. Although we can correct the upper bound of $\left|\left(Q_{m}-I_{m}\right)_{i, j}\right|$ by deriving a similar upper bound for $i>j$ as $i \leq j$, the error regarding the transformation into an upper Hessenberg matrix is crucial for deriving Proposition 3.1. However, we can derive the same conclusion as Theorem 3.1 by modifying the assumptions of Theorem 3.1 and Proposition 3.1. To show the modified version of Proposition 3.1, we do not need Householder reflectors. Instead, we use Cauchy's integral formula to show it. Correct statements and proofs of Theorem 3.1

The original article can be found online at https://doi.org/10.1007/s10543-020-00829-w.

Yuka Hashimoto

yukahashimoto@math.keio.ac.jp

Takashi Nodera

nodera@math.keio.ac.jp

1 Graduate School of Science and Technology, Keio University, 3-14-1 Hiyoshi, Kohoku, Yokohama, Kanagawa 223-8522, Japan

2 Faculty of Science and Technology, Keio University, 3-14-1 Hiyoshi, Kohoku, Yokohama, Kanagawa 223-8522, Japan 
and Proposition 3.1 are as follows. Here, in addition, we reorganize the statements of Theorem 3.1 and Proposition 3.1 to clarify the dependence of each variable.

We define $\tilde{H}_{m}:=T_{m}-H_{m} D_{m}+\gamma_{m} H_{m}$ and $L_{m}(z):=\left(z H_{m}-\tilde{H}_{m}\right)^{-1}$ for $z \in \mathbb{C}$. Note that $z H_{m}-\tilde{H}_{m}$ is an upper Hessenberg matrix and thus, $L_{m}(z)$ is the inverse of an upper Hessenberg matrix. To show the theorem, we require the following assumption:

Assumption 3.1 Let $\hat{\alpha}>0$ and $0<\lambda<1$ be given constants and let $\Pi \subseteq \mathbb{C}$ be a given bounded open set. We assume for any $z \in \partial \Pi$

$$
\begin{aligned}
& \left|\left(L_{m}(z)\right)_{i, j}\right| \leq \hat{\alpha} \lambda^{i-j} \quad(i \geq j), \\
& \Lambda\left(K_{m}^{-1}\right) \subseteq \Pi,
\end{aligned}
$$

where $\Lambda\left(K_{m}^{-1}\right)$ is the spectrum of the matrix $K_{m}^{-1}$.

Theorem 3.1 Let $\hat{\alpha}>0,0<\lambda<1$, and $\delta>0$ be constants and let $\Pi \subseteq \mathbb{C}^{+}$be $a$ bounded open set whose boundary is a rectifiable Jordan curve oriented in positive sense. If the matrices $L_{m}(z)$ and $K_{m}$ satisfy Assumption 3.1 with $\hat{\alpha}, \lambda$, and $\Pi$, and if the residual of solving the linear equation $\left(\gamma_{m} I-A\right) x_{m}=V_{m} t_{m}$ satisfies $\left\|r_{m}^{\mathrm{sys}}\right\| \leq \delta$, then the first term of the Eq. (3.4) is bounded as

$$
\begin{aligned}
& \beta\left|h_{m+1, m} e_{m}^{*} \phi_{k}\left(D_{m}-H_{m}^{-1} T_{m}\right) H_{m}^{-1} e_{1}\right|\left\|\left(\gamma_{m} I-A\right) v_{m+1}\right\| \\
& \leq \beta(1+\delta) \kappa\left(\gamma_{m} I-A\right) \alpha \lambda^{m-1}
\end{aligned}
$$

where $\alpha=2 \pi^{-1}|\partial \Pi| \phi_{k}(N) \hat{\alpha}$ and $|\partial \Pi|=\int_{\partial \Pi}|d z|$.

Moreover, for any tolerance tol ${ }_{\phi}>0$ for approximating the vector $\phi_{k}(A) v$ and for any $m^{\max }>0$, if $m \leq m^{\max }$ and if

$$
\begin{aligned}
\left\|r_{1}^{\mathrm{sys}}\right\| & \leq \frac{\operatorname{tol}_{\phi}}{m^{\max } \beta\left\|\phi_{k}\left(D_{m}-H_{m}^{-1} T_{m}\right) H_{m}^{-1} e_{1}\right\|}, \\
\left\|r_{j}^{\mathrm{sys}}\right\| & \leq \frac{\left|g_{1,1}^{m}\right| \lambda}{\left|g_{j, 1}^{m}\right|}\left\|r_{1}^{\mathrm{sys}}\right\| \quad(j=2, \ldots, m),
\end{aligned}
$$

then the second term of Eq. (3.4) can be evaluated as

$$
\beta\left\|R_{m}^{\mathrm{sys}} \phi_{k}\left(D_{m}-H_{m}^{-1} T_{m}\right) H_{m}^{-1} e_{1}\right\| \leq \operatorname{tol}_{\phi},
$$

where $g_{i, j}^{m}=\left(\phi_{k}\left(D_{m}-H_{m}^{-1} T_{m}\right) H_{m}^{-1}\right)_{i, j}$.

Proposition 3.1 Let $\hat{\alpha}>0,0<\lambda<1$ be constants and let $\Pi \subseteq \mathbb{C}^{+}$be a bounded open set whose boundary is a rectifiable Jordan curve oriented in positive sense. If the matrices $L_{m}(z)$ and $K_{m}$ satisfy Assumption 3.1 with $\hat{\alpha}, \lambda$, and $\Pi$, then we have

$$
\left|\left(\phi_{k}\left(D_{m}-H_{m}^{-1} T_{m}\right) H_{m}^{-1}\right)_{i, j}\right| \leq \frac{1}{2 \pi}|\partial \Pi| \phi_{k}(N) \hat{\alpha} \lambda^{i-j} \quad(i \geq j) .
$$


Proof Since $\phi_{k}$ is an entire function, by Cauchy's integral formula, we have

$$
\begin{gathered}
\phi_{k}\left(D_{m}-H_{m}^{-1} T_{m}\right) H_{m}^{-1}=H_{m}^{-1} \phi_{k}\left(\gamma_{m} I-K_{m}^{-1}\right)=\frac{1}{2 \pi \mathrm{i}} H_{m}^{-1} \int_{\partial \Pi} \phi_{k}\left(\gamma_{m}-z\right)\left(z I-K_{m}^{-1}\right)^{-1} d z \\
=\frac{1}{2 \pi \mathrm{i}} H_{m}^{-1} \int_{\partial \Pi} \phi_{k}\left(\gamma_{m}-z\right) H_{m}\left(z H_{m}-\tilde{H}_{m}\right)^{-1} d z=\frac{1}{2 \pi \mathrm{i}} \int_{\partial \Pi} \phi_{k}\left(\gamma_{m}-z\right) L_{m}(z) d z .
\end{gathered}
$$

Moreover, for $i \geq j$, we have

$$
\begin{gathered}
\left|\left(\frac{1}{2 \pi \mathrm{i}} \int_{\partial \Pi} \phi_{k}\left(\gamma_{m}-z\right) L_{m}(z) d z\right)_{i, j}\right| \leq \frac{1}{2 \pi} \int_{\partial \Pi}\left|\phi_{k}\left(\gamma_{m}-z\right)\right||d z| \hat{\alpha} \lambda^{i-j} \\
\leq \frac{1}{2 \pi}|\partial \Pi| \max _{z \in \partial \Pi} \phi_{k}\left(\gamma_{m}-\operatorname{Re}(z)\right) \hat{\alpha} \lambda^{i-j} \leq \frac{1}{2 \pi}|\partial \Pi| \phi_{k}(N) \hat{\alpha} \lambda^{i-j},
\end{gathered}
$$

where $\operatorname{Re}(z)$ is the real part of $z$. The second inequality holds since $\phi_{k}$ is represented as $\phi_{k}(z)=\int_{0}^{1} e^{(1-s) z} \frac{s^{k-1}}{(k-1) !} d s$ and the last inequality holds since $N \geq \gamma_{m}$ for any $m<N / h$. This completes the proof of Proposition 3.1.

The modified version of Theorem 3.1 is derived by Eq. (3.14) in the same manner as the proof in the original article.

Remark 3.2 If $z H_{m}-\tilde{H}_{m}$ is diagonalizable and invertible, there exist constants $\hat{\alpha}_{m}(z)>0$ and $0<\lambda_{m}(z)<1$ such that $\left|\left(L_{m}(z)\right)_{i, j}\right| \leq \hat{\alpha}_{m}(z) \lambda_{m}(z)^{i-j}$. The first assumption about $L_{m}(z)$ in Assumption 3.1 is about the uniformity of $\hat{\alpha}_{m}(z)$ and $\lambda_{m}(z)$. Indeed, let $P_{m}(z) \Delta_{m}(z) P_{m}(z)^{-1}$ be an eigenvalue decomposition of $z H_{m}-\tilde{H}_{m}$ and let $\Sigma_{m}(z) \subseteq \mathbb{C} \backslash\{0\}$ be a bounded open set whose boundary is a rectifiable Jordan curve oriented in positive sense such that $\Lambda\left(z H_{m}-\tilde{H}_{m}\right) \subseteq \Sigma_{m}(z)$. Since $z H_{m}-\tilde{H}_{m}$ is an upper Hessenberg matrix, for $i>j$, any polynomial $p \in \mathcal{P}_{i-j-1}$ satisfies

$$
\begin{aligned}
\left|\left(L_{m}(z)\right)_{i, j}\right| & =\left|\left(L_{m}(z)\right)_{i, j}-\left(p\left(z H_{m}-\tilde{H}_{m}\right)\right)_{i, j}\right| \leq\left\|L_{m}(z)-p\left(z H_{m}-\tilde{H}_{m}\right)\right\| \\
& \leq\left\|P_{m}(z)\right\| \sup _{w \in \Sigma_{m}(z)}\left|w^{-1}-p(w)\right|\left\|P_{m}(z)^{-1}\right\| .
\end{aligned}
$$

Let $f(w):=w^{-1}$. We set the polynomial $p$ as the truncated Faber series of $f[1$, Section 2]. Then by Corollary 2.2 in Ellacott [1], there exist constants $C_{m}(z)>0$ and $0<\lambda_{m}(z)<1$ such that

$$
\sup _{w \in \Sigma_{m}(z)}\left|w^{-1}-p(w)\right| \leq C_{m}(z) \lambda_{m}(z)^{i-j}
$$

Thus, we have $\left|\left(L_{m}(z)\right)_{i, j}\right| \leq \kappa\left(P_{m}(z)\right) C_{m}(z) \lambda_{m}(z)^{i-j}$.

If $\Sigma_{m}(z)$ is independent of $m$ and $z$, then $C_{m}(z)$ and $\lambda_{m}(z)$ are independent of $m$ and $z$. Therefore, if in addition there exist a constant $\tilde{\alpha}>0$ such that $\kappa\left(P_{m}(z)\right) \leq \tilde{\alpha}$, then the first assumption about $L_{m}(z)$ in Assumption 3.1 is satisfied.

The second assumption about $K_{m}$ in Assumption 3.1 is satisfied if there exists a bounded open set $\Sigma \subseteq \mathbb{C}^{+}$such that $W\left(\left(\gamma_{m} I-A\right)^{-1}\right) \subseteq \Sigma$ and if $f_{j}^{\text {sys }}=\mathbf{0}$ for 
$j=1, \ldots, m$, that is, the linear equations solved exactly. Indeed, by Eq. (3.2), the identity $K_{m}=V_{m}^{*}\left(\gamma_{m} I-A\right)^{-1} V_{m}$ holds in this case. Therefore, we have $\Lambda\left(K_{m}\right) \subseteq$ $W\left(K_{m}\right) \subseteq W\left(\left(\gamma_{m} I-A\right)^{-1}\right) \subseteq \Sigma$.

\section{Typos}

1. Before Eq. (1.1), " $u(\cdot, x) \in C(0, T)$ for all $x \in \Omega$ " should read " $u \in$ $C\left([0, T], L^{2}(\Omega)\right)$ ".

2. In Eqs. (1.4) and (1.5) and the formula between them, $g(y(s)), g_{l}$, and $g_{i-1}$ should read $M^{-1} g(y(s)), M^{-1} g_{l}$, and $M^{-1} g_{i-1}$, respectively.

3. The sum $\sum_{k=1}^{r-1}$ in Eq. (1.7) should read $\sum_{k=0}^{r}$.

4. Eq. (2.5) should read $t_{j}=e_{\rho\lfloor(j-1) / \rho\rfloor+1} \in \mathbb{R}^{j}$.

5. In the middle of the proof of Theorem 3.1, $\left(\gamma_{m} I-A\right)^{-1} v_{m}-f_{m}^{\text {sys }}$ should read $\left(\gamma_{m} I-A\right)^{-1} V_{m} t_{m}-f_{m}^{\text {sys }}$.

6. Eq. (3.15) should read

$$
\begin{aligned}
& \beta\left\|H_{m}^{-1} \phi_{k}\left(\left(H_{m} D_{m}-T_{m}\right) H_{m}^{-1}\right) e_{1}\right\| \approx \beta\left\|H_{m}^{-1}\left[\phi_{k}\left(\left(H_{m} D_{m}-T_{m}\right) H_{m}^{-1}\right)\right]_{1,1} e_{1}\right\| \\
& \quad \approx \beta\left\|V_{m}^{*}\left(\gamma_{1} I-A\right) V_{m} \phi_{k}\left(\left(H_{m} D_{m}-T_{m}\right) H_{m}^{-1}\right) e_{1}\right\| \\
& \quad \approx\left\|\left(\gamma_{1} I-A\right) y(t)\right\| \approx\left\|\left(\gamma_{1} I-A\right) y(0)\right\| .
\end{aligned}
$$

Moreover, the formula about tol ${ }_{1}^{\text {sys }}$ in the last paragraph in Section 3 should read tol $_{1}^{\text {sys }}=\operatorname{tol}_{\phi} /\left[m^{\max }\left\|\left(\gamma_{1} I-A\right) y(0)\right\|\right]$.

7. In the last paragraph in Remark 3.1 , the definition of $j_{0}$ should read $j_{0}:=\rho\lfloor(j-$ $1) / \rho\rfloor+1$. Moreover, we need an additional assumption $\left|g_{j_{0}, 1}^{m}\right|\left(j_{0}-1\right) /\left(j_{0}+1\right) \geq$ $\left|g_{j, 1}^{m}\right|(j-1) /(j+1)$ for deriving the last formula in Remark 3.1.

8. At the beginning of Example 2, $(-1.5,1.5) \times(-1,1)$ should read $\Omega=$ $(-1.5,1.5) \times(-1,1)$.

9. Eq. (4.2) should read

$$
\left\{\begin{array}{l}
\tilde{M} \ddot{\tilde{y}}(t)=\tilde{L} \tilde{y}(t)+\tilde{b}(t), \\
\tilde{y}(0)=\tilde{v}, \quad \dot{\tilde{y}}(0)=\mathbf{0} .
\end{array}\right.
$$

10. In Example 3, $\partial \Omega$ in the boundary condition " $u=0, v=0$ on $(0, T] \times \partial \Omega$ " should read $\partial \Omega_{1}$ and $\partial \Omega$ in " $\frac{\partial u}{\partial \mathbf{n}}=0, \frac{\partial v}{\partial \mathbf{n}}=0$ on $(0, T] \times \partial \Omega$ " should read $\partial \Omega_{2}$, where $\partial \Omega_{1}=[-1.5,1.5] \times\{1,-1\}$ and $\partial \Omega_{2}=\partial \Omega \backslash \partial \Omega_{1}$.

11. In Example 3, the formula $g_{i}(y)=F(y)-L_{i-1} y=Q(y) y-Q\left(y_{i-1}\right) y$ should read $g_{i}(y)=F(y)-L_{i} y=Q(y) y-Q\left(y_{i}\right) y$ and the scheme of the exponential integrator should read $y_{i+1}=y_{i}+\Delta t \phi_{1}\left(\Delta t M^{-1} L_{i}\right) M^{-1} F\left(y_{i}\right)-\Delta t \frac{2}{3} \phi_{2}\left(\Delta t M^{-1} L_{i}\right) M^{-1}\left(g_{i}\left(y_{i}\right)-g_{i}\left(y_{i-1}\right)\right)$.

In addition, the formula $\phi_{2}\left(\Delta t M^{-1} L_{i}\right)\left(g_{i}\left(u_{i}\right)-g_{i}\left(u_{i-1}\right)\right)$ written after the scheme should read $\phi_{2}\left(\Delta t M^{-1} L_{i}\right) M^{-1}\left(g_{i}\left(y_{i}\right)-g_{i}\left(y_{i-1}\right)\right)$. 
Acknowledgements We would like to thank Prof. Tatsuo Iguchi for pointing out the errors to us.

\section{Reference}

1. Ellacott, S.W.: Computation of Faber series with application to numerical polynomial approximation in the complex plane. Math. Comput. 40(162), 575-587 (1983)

Publisher's Note Springer Nature remains neutral with regard to jurisdictional claims in published maps and institutional affiliations. 